\title{
Futuristic Smart Interactive E-Speculum
}

\author{
Harish Bakker ${ }^{1}$, Jishnu $\mathrm{S}^{2}$, Musthafa $\mathbf{N}^{3}$, K Neethu Sathyan ${ }^{4}$, Shahaziya Parvez $\mathbf{M}^{5}$ \\ UG Scholar, Applied Electronics and Instrumentation, IES College of Engineering, Thrissur, India ${ }^{1,2,3}$ \\ Asst. Professor, Applied Electronics and Instrumentation, IES College of Engineering, Thrissur, India ${ }^{4,5}$
}

\begin{abstract}
This paper describes the design and development of a futuristic interactive mirror which can be placed in the washroom that presents an unobtrusive interface for the ambient washroom environment. The mirror provides natural means of interaction through which the user is able to do some control mechanisms. The mirror displays information's like date, time, day of the week, water level in the overhead tank and temperature of the water in the pipeline. The control mechanism includes the control of water level, temperature and removal of fog raised on the surface of the mirror. Emphasis is also given to ensure convenience in accessing these services with a simple user interface. A service-oriented architecture has been adopted to develop and deploy the various functionalities like display and Corresponding control mechanism in both manual and automatic mode.
\end{abstract}

Keywords: Liquid crystal display, pyro electric infrared sensor, Infrared, Analog to digital converter, Real time clock, thin film transistor, single pole dual throw.

\section{I.INTRODUCTION}

The technology is making headway, and inventions and objects seen in futuristic movies become reality in a short time, many of the things we thought impossible a few years ago are now the objects used in daily life. We are sure that a large portion of our busy at-home lives are spent in kitchens and bathrooms. This unique product offers the ability to extend your everyday computing access to a mirror surface with multi-touch control. The technology to do all of this is already here even though it's still pricey right now. But with the prices of displays and smart components dropping by the day, interactive touch screen mirrors aren't far away from our everyday life. Say for example, the mirror had your news, or stocks, or twitter displayed...that time spent in front of the mirror, whether you're getting your make-up or hair done, or just brushing your teeth, would be more useful. The mirror could have a full web browser, along with loads of useful apps (weather, YouTube, emails etc.) and maybe a few games installed on it as well, for all you know, it could be a full tablet-like experience.

A mirror that responds to your presence, a mirror that is built to improve your daily productivity. The Smart Interactive Mirror helps you stay organized right from the start of the day. The time when you stand in front of the mirror while brushing your teeth or arranging your hair, the mirror detects your presence and provides you with the latest news, the schedule of the day and the weather conditions for your area. The idea for this project was got from a book named "Physics of the future" by michiokaku which narrates about the future of the world, in which the author tells us that in the future also the first thing that we are going to do when we get up from bed in the morning is to face a mirror. So just imagine that the mirror that we are going to face in the future morning is an intelligent. Our locally designed mirror for bathrooms enables the end user to conveniently access the water level, temperature, date, time etc. These specialized touch screen mirrors are the new frontier of the bathroom and kitchens.

We can place this mirror in the following areas of our own house like the living room, bathroom, and kitchen. We are here by specifying the use of this mirror when it is placed in the bathroom. The condition when it is placed in living room and kitchen can be viewed as an extension to our project. The location of the touch display and the size of the mirror are Customizable.

Here in this project we are incorporating four different modules for special functions.

- Defogger

- Date and Time Information

- Water Level sensor

- Temperature Sensor

\section{II.PROPOSED SYSTEM}

The block diagram of the system contains four different parts. They are the core, input, output and the user interface section. The core of the system is the microcontroller which is Atmel - mega 2560. It is a 100 pin IC with inbuilt analog to digital converter. The core creates a simple interface between the input section output section and the user interface. 
Vol. 5, Issue 4, April 2017

When the signals are received from the input section and the user interface section the controller processes the data and corresponding output is produced which is either displayed through the screen or operates the drivers.

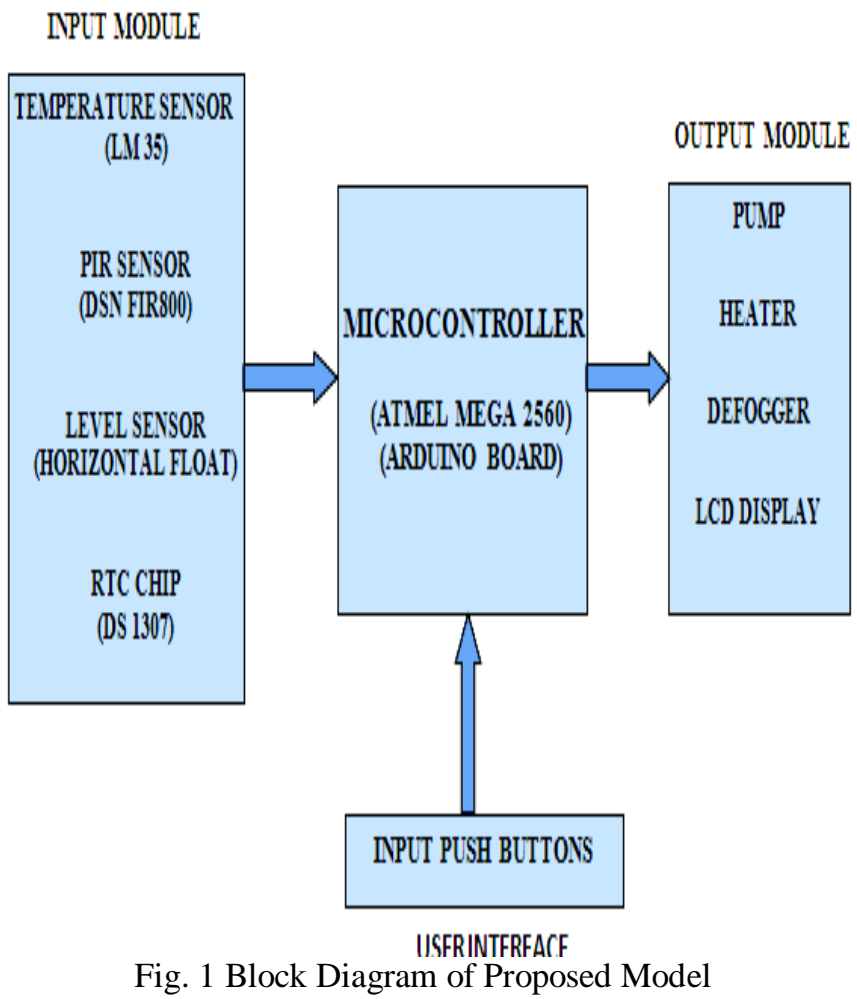

The input section contains sensors and IC's of various functions incorporated to do various sensing actions need in the proper functioning of the system. The major sensors used are LM35 for temperature sensing, DSN FIR800 which is a pyro electric infrared sensor used for motion detection and a Horizontal float switch used for level detection. The IC used in the input section is DS1307 which is a real time clock chip. They will be discussed in details in the following sections.

The output section of the system contains drivers like pump, heater and motor fan and a 2.4" TFT LCD display. All the drivers a operated at $12 \mathrm{v}$ DC power received from the power supply circuit. The TFT display increases the aesthetics of the system with improved display quality and contrast. The user interface sections contain only switches which are provided for the manual control and operation of the functionalities inside the bathroom.

\section{III.PRINCIPLE OF OPERATION}

\section{A. POWER SUPPLY}

The whole power for the working of this devise is obtained from the switch placed outside the door of the bathroom which if fed with 230v supply. Since the controller (Atmel mega 2560) of the device and other components operates at $+5 \mathrm{v}$ and $+12 \mathrm{v}$ respectively, it is necessary to convert the AC supply in to DC supply and regulate it to $+5 \mathrm{v}$ and $+12 \mathrm{v}$. A full bridge rectifier circuit with four diodes $1 \mathrm{~N} 4007$ is used for the purpose of rectification. Followed by this arrangement the voltage regulator IC's LM7805 and LM7812 are connected in parallel for two output voltages. When the switch is on the device will be fed with that voltage that it requires to go on. When the voltage is received to the terminals of the components like controller and sensors it gets active, thus the controller starts working and the sensors will give the needed inputs to the controller.

\section{B. IR LOCK}

The device contains an IR lock and unlocks system for efficient using of electricity. That is even though the switch at the door is on; the device will start displaying the parameters only when the IR sensor output is reached to the controller. It means this that the display will be generated only when a person is there in front of the mirror and will stay on for a particular time. This system is required because the display which is a 2.4" TFT LCD needs more power to operate. It is similar to the smart eye setup used in the recently launched smart phones. The sensor used for this purpose is DSN-FIR800 PIR Sensor. 


\section{WATER LEVEL CONTROL}

The main purpose of the proposed project is to display out the level of the water present in the overhead water tank so as to help people from being stuck in the bathroom because of low water level conditions and related problems. Here the water level in the tank is calculated using the float switches fixed at three different levels of the tank. The float switches are at normally off state. They are turned on when the water level rises over them. They have two terminals. One of the terminals if fed with a $+5 \mathrm{v}$ supply and the other terminals gives the output. When the switch is open, no signal will be reached to the controller and when the switch is closed, signal will be reached to the controller. This makes a binary low and high condition. The first switch corresponds to level of $25 \%$ water. The second one corresponds to a level of $50 \%$ and the third one corresponds to level of $98 \%$. An allowance of $2 \%$ is provided to eliminate the overflow condition.

Here the controller have the duty of calculating the level of water with the received data and it is also responsible for the automatic switch on of the pump if the water level of the tank is below $25 \%$ that to only when there is a person inside the bathroom. The user is required to switch off the pump even if the pump is switched on automatically and if there is no input given by the user to the device about the level at which the pump should stop pumping water using the touch pad provided. Even the level of water is above $25 \%$ and the user need more water then he can switch on the pump by himself. A push button switch is provided for this purpose. The same switch is used for switching off the pump. There are two outputs for this setup. A display and the circuit of SPDT switch and pump. The level of water in the tank will be shown through the display mounted on the back of the mirror. Seeing this output one can decide whether to start or stop the pump.

\section{WATER TEMPERATURE DETECTION}

Another important information to be displayed using this device is the level of heat of the water in the tank. For this purpose the sensor used is LM35. The mentioned sensor LM35 is a device that provides an analog output. The controller has got the ability to convert the analog input to digital input using the inbuilt ADC. The sensor is place in the water tank by using a particular waterproof structure. The sensor LM35 has got three pins. One of the pins (pin 1) is provided with $+5 v$ supply. Pin 2 gives the output. Pin 3 is connected to the ground.

This setup is used to let the user inform that the temperature level of the water tank is at a particular level. So that the user can decide whether he has to heat the water up or use the water in the tank with the same heat level. Here the controller have the duty of calculating the temperature of water with the received data but it is not responsible for the automatic switch on of the heater even if the water temperature of the tank is below 20 degree Celsius. The user is required to switch on and off the heater manually using the push button switch.There are two outputs for this setup. A display and a circuit of SPDT switch and heater. The temperature of water in the tank will be shown through the display mounted on the back of the mirror. Seeing this output one can decide whether to start or stop the heater.

\section{E. DEFOGGER}

This setup does not use a sensor. When fog arises on the surface of the mirror the user can switch on the fan to remove the fog by using the push button switch. The fan can be switched off using the same switch when the fog is completely removed or removed to that particular extends which we require.

\section{F. REAL TIME CLOCK}

This system contains an IC named DS1307 an 8 pin IC that which provides the display of date, time and day of the week when interfaced with a controller and a display. It is with the help of the crystal used for oscillation that the chip is able to give outputs of RTC. The crystal used for providing oscillations is of the rating $32.768 \mathrm{KHz}$. To avoid the frequent resetting of the data a battery backup of $3 \mathrm{v}$ is provided with a lithium ion button battery which will provide uninterrupted supply to the chip.

\section{ADVANTAGES \& DISADVANTAGES.}

A. Advantages

The following are the advantages of the proposed system:

- Less space consumption.

- Information about the water level and temperature will alert the user.

- Automatic defogger helps the user from extra effort for cleaning the mirror.

- Multipurpose.

- Customizable. 


\section{IJIREEICE \\ International Journal of Innovative Research in Electrical, Electronics, Instrumentation and Control Engineering \\ ISO 3297:2007 Certified \\ Vol. 5, Issue 4, April 2017}

B. Disadvantages

The system faces some demerits like:

- Expensive

- Mirror casing must be water proof.

- Requires constant power supply.

\section{RESULTS \& DISCUSSION}

The design of the mirror was done successfully. The mirror was able to display out the information's like date, time and day of the week, level of the water in overhead tank and the temperature of the water in the pipeline. The control mechanisms which the mirror was intended to do with the driving circuits of the pump, heater and the defogger was established and proper controlling was obtained. The output of the level and temperature sensors were obtained with precision.

The figure 2 given below shows the display of the system. The system was able to show response to every change in the level and temperature as expected.

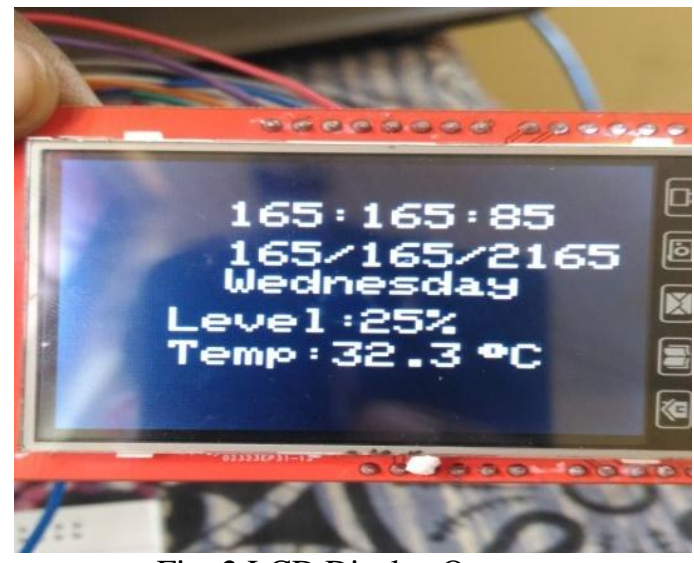

Fig. 2 LCD Display Output.

\section{CONCLUSION}

In this paper we have successfully designed and setup a mirror that can be placed in the washroom which displays information like time, date, day, water level, water temperature and controls some parameters manually and automatically. The location of the touch display and the size of the mirror are customizable.

As an extension in future, the following characteristics can be added.

- Interconnection with smartphones.

- Interfacing biosensors and calorimeters.

- Voice based action and response.

- On board camera for better shaving.

\section{REFERENCES}

[1] "Physics of the Future" by MachioKaku.

[2] Jun-Ren Ding, Chien-Lin Huang, Jin-Kun Lin, Jar-Ferr Yang, and Chung- Hsien Wu, "Magic Mirror," IEEE International Symposium on Multimedia, December 2007, pp. 176-185

[3] Tatiana Lashina, "Intelligent Bathroom," Philips Research, Netherlands.

[4] www.adafruit.com/smart_mirror.

[5] Carlos Hitoshi Morimoto. "Interactive Digital Mirror,” IEEE Proceedings on Computer Graphics and Image Processing, 2001 , pp. $232-236$.

\section{BIOGRAPHIES}

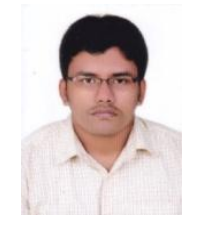

Harish Bakker currently pursuing the Bachelor's degree in Applied Electronics \& Instrumentation Engineering from the IES College of Engineering, Thrissur. 
Vol. 5, Issue 4, April 2017

Jishnu S currently pursuing the Bachelor's degree in Applied Electronics \& Instrumentation Engineering from the IES College of Engineering, Thrissur.

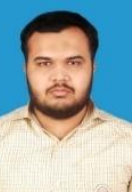

Musthafa N currently pursuing the Bachelor's degree in Applied Electronics \& Instrumentation Engineering from the IES College of Engineering, Thrissur.

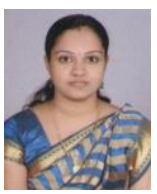

K Neethu Sathyan completed Master's Degree in Instrumentation Engineering from RVS College of Engineering \& Technology, Dindigul. She is currently working as Assistant Professor in Department of Electronics and Communication Engineering, IES College of Engineering, Thrissur.

Shahaziya Parvez. M, received the Bachelor's degree from the Anna University-Coimbatore and the M.Tech. Degree in from Mahatma Gandhi University, Kottayam. She is currently working as an Assistant Professor at IES College of Engineering, Thrissur, Kerala, India. 